\title{
Avaliação do meio escolar: percepções dos profissionais de uma escola pública do interior de São Paulo
}

\author{
Jaqueline Stefanini, Luciana Cavalin, \\ Débora Silva, Natália Camargo, \\ Ana Sani, Edson Scherer \& Zeyne Scherer
}

Resumo:

Este estudo objetivou conhecer a percepção dos profissionais de uma escola da rede pública estadual de educação básica do interior do Estado de São Paulo, sobre o meio escolar onde desempenham suas funções. Trata-se de um estudo qualitativo, do tipo exploratório e descritivo, em que os dados foram coletados a partir de um questionário, numa amostra de 21 profissionais, com idades entre 20 e 60 anos. Os resultados mostram que apesar da percepção positiva em relação a estrutura física da escola, há falta de recursos financeiro que afetam o seu funcionamento. O envolvimento dos pais é negativo e existem comportamentos problemáticos na escola que exigem o envolvimento de todos os atores escolares e equipe multidisciplinar para melhorar o sistema disciplinar da escola. Conclui-se que a avaliação do meio escolar pode contribuir para o desenvolvimento de ações intersetoriais, tendo como propósito a prevenção e enfrentamento dos comportamentos problemáticos dos estudantes e a violência.

Palavras-chave:

ambiente escolar; comportamentos; avaliação. 


\title{
Evaluation of the school environment: perceptions of the professionals of a public school
}

\begin{abstract}
This study aimed to know the perception of the professionals of a school in the state public basic education network in the interior of the State of São Paulo, about the school environment in which they perform their functions. This is a qualitative, exploratory and descriptive study, in which the data were collected from a questionnaire in a sample of 21 professionals, aged between 20 and 60 years. The results show that despite the positive perception regarding the physical structure of the school, there is a lack of financial resources that affect its functioning. Parent involvement is negative and there are problematic behaviors at school that require the involvement of all school actors and multidisciplinary team to improve the school's disciplinary system. It is concluded that the evaluation of the school environment can contribute to the development of intersectoral actions, with the purpose of preventing and coping with students' problematic behaviors and violence.
\end{abstract}

Keywords: school environment; behaviors; evaluation.

\section{Evaluation de l'environnement scolaire: perceptions des professionnels d'une école publique}

Résumé: Cette étude visait à connaître la perception des professionnels d'une école dans le réseau public d'éducation de base publique de l'intérieur de l'État de São Paulo, sur l'environnement scolaire dans lequel ils exercent leurs fonctions. II s'agit d'une étude qualitative, exploratoire et descriptive, dans laquelle les données ont été collectées à partir d'un questionnaire, auprès d'un échantillon de 21 professionnels, âgés de 20 à 60 ans. Les résultats montrent que malgré la perception positive concernant la structure physique de l'école, il y a un manque de ressources financières qui affectent son fonctionnement. La participation des parents est négative et il y a des comportements problématiques à l'école qui nécessitent la participation de tous les acteurs de l'école et de l'équipe multidisciplinaire pour améliorer le système disciplinaire de l'école. II est conclu que l'évaluation de l'environnement scolaire peut contribuer au développement d'actions intersectorielles, dans le but de prévenir et de faire face aux comportements problématiques et à la violence des élèves.

Mots-clés: environnement scolaire; comportements; évaluation.

\section{Evaluación del entorno escolar: percepciones de los profesionales de una escuela pública}

Resumen: Este estudio objetivó conocer la percepción de los profesionales de una escuela de la red pública estatal de educación básica del interior del Estado de São Paulo, sobre el medio escolar donde desempeñan sus funciones. Se trató de un estudio cualitativo, de tipo exploratorio y descriptivo, en que los datos fueron recolectados a partir de un cuestionario, en una muestra de 21 profesionales, con edades entre 20 y 60 años. Los resultados muestran que a pesar de la percepción positiva en relación a la estructura física de la escuela, hay falta de recursos financieros que afectan su funcionamiento. La participación de los padres es negativa y hay comportamientos problemáticos en la escuela que exigen la participación de todos los actores escolares y equipo multidisciplinario para mejorar el sistema disciplinario de la escuela. Se concluye que la evaluación del medio escolar puede contribuir al desarrollo de acciones intersectoriales, teniendo como propósito la prevención y enfrentamiento de los comportamientos problemáticos de los estudiantes y la violencia.

Palabras clave: ambiente escolar; comportamiento; evaluación. 


\section{Introdução}

A escola tem um papel formal relevante e integrativo na socialização dos indivíduos, sendo igualmente determinante para o seu desenvolvimento psicossocial (Brenner \& Carrano, 2014). No entanto, o ambiente escolar pode afetar ambos os processos, dependendo da maneira como a instituição lida com os problemas e conflitos instalados nesse contexto.

No Brasil as escolas públicas têm enfrentado desafios de natureza multidimensional, envolvendo a diversidade dos atores escolares, formação inadequada dos profissionais, sobrecarga do trabalho docente, precária estrutura física das escolas, diminuição do investimento na educação pública, desigualdades escolares entre sistemas e redes de ensino, dentre outros problemas que que afetam a rotina escolar (Brenner \& Carrano, 2014). A escola localizada em região vulnerável, do ponto de vista econômico e social, também pode enfrentar dificuldades relacionadas à violência urbana, incluindo o tráfico de drogas.

Nesse contexto, realizar estudos sobre as especificidades da instituição, incluindo a sua estrutura, o seu funcionamento, as características e comportamentos dos estudantes, é relevante, pois, quanto mais informações forem obtidas sobre a instituição escolar, melhor será a qualidade das ações desenvolvidas em benefício da escola e sua comunidade (Nunes, Caridade, \& Sani, 2015).

\section{Desafios enfrentados pelas escolas brasileiras}

No Brasil, a Lei de Diretrizes e Bases da Educação Nacional (LDB) garante a oferta de educação escolar regular para jovens e adultos, e assegura a Educação Básica obrigatória e gratuita dos 4 aos 17 anos de idade para todos os cidadãos brasileiros (Brasil, 2013). A educação é, portanto, um direito social e dever do Estado que além de garantir vagas deve oferecer condições para permanência do estudante na escola. Desse modo, é importante considerar a diversidade e extensão do território brasileiro, incluindo os aspectos de vulnerabilidade social e econômica que afetam a rotina escolar (Érnica \& Batista, 2012).

Estudo realizado na região de São Paulo com 400 mil pessoas, sobre a influência do território no desenvolvimento de problemas educacionais, evidenciou que crianças e jovens permanecem matriculados, mas as escolas não asseguram a proteção e as condições de aprendizado e desenvolvimento. Os resultados mostram também que quanto maior a vulnerabilidade social menor são as oportunidades educacionais oferecidas aos estudantes, além disso, o sistema escolar reproduz a segregação social na composição do corpo discente do sistema de ensino público paulistano (Érnica \& Batista, 2012). 
Em diferentes regiões do Brasil as escolas públicas têm enfrentado dificuldades como a superlotação de salas de aula, as condições precárias de trabalho dos professores, incluindo a carga horária e remuneração, as características sociais e econômicas do entorno da escola e a violência urbana presente nesse ambiente, tais dificuldades afetam o processo de ensino-aprendizagem e, igualmente, a relação professor-estudante (Becker \& Kassouf, 2016; Bernardes, 2015; Érnica \& Batista, 2012; Monteiro \& Da Silva, 2015).

A escola é um espaço de interação social, ambiente que influencia os comportamentos de quem o frequenta e é influenciado por esses comportamentos, origina percepções especificas sobre o ambiente e seu funcionamento, tornando-se um contexto de divergências onde os profissionais apresentam dificuldades para gerenciar tais circunstâncias. Essas dificuldades podem contribuir para o desenvolvimento de comportamentos inadequados no meio escolar como o desrespeito generalizado, não cumprimento de regras estabelecidas pela instituição, incivilidades, uso da violência e depredação do patrimônio, mesmo tendo favoráveis as condições físicas da escola e de seu entorno (Nunes et al., 2015).

Dada a complexidade, multiplicidade e dificuldades que permeiam as escolas brasileiras, é importante conhecer as características desse ambiente e seu entorno, o funcionamento escolar e os comportamentos dos estudantes, pois, assim pode-se mapear os principais problemas da escola a fim de planejar e implementar ações que possam melhorar o funcionamento da instituição e, consequentemente, promover o desenvolvimento de seus estudantes (Nunes et al., 2015). No presente estudo, buscou-se conhecer a percepção dos profissionais de uma escola da rede pública estadual de educação básica do interior do Estado de São Paulo, sobre o meio escolar onde desempenham suas funções para a avaliação desta instituição.

\section{Método}

Trata-se de um estudo de natureza qualitativa, do tipo exploratório e descritivo (Ollaik \& Ziller, 2012), em que serão apresentados os resultados acerca das percepções dos profissionais de uma escola de educação básica brasileira sobre o meio escolar e seu entorno, o funcionamento interno e externo, as caraterísticas e os comportamentos dos estudantes que frequentam essa escola.

\section{Participantes}

A escola objeto deste estudo contava com 43 funcionários e atendia um total de 680 estudantes, na faixa etária de 13 a 20 anos, do ensino fundamental 2 e do ensino médio. A instituição está localizada em território vulnerável, do ponto de vista social e econômico, 
possui frequentes situações de violência e dificuldades na condução do processo ensino-aprendizagem (Scherer, Scherer, Cocenas, \& Gregorio, 2011). Essa instituição dispõe ainda de um professor mediador escolar e comunitário (PMEC) responsável exclusivamente pela mediação de conflitos no ambiente da escola (Martins \& Alves, 2018).

Participaram voluntariamente 21 profissionais que demonstraram disponibilidade e interesse, sendo 13 do sexo feminino e 8 do sexo masculino. Quanto a escolaridade, houve sobretudo a participação de profissionais com ensino superior completo (18), exercendo a função de professor (15) (cf. Tabela 1).

Tabela 1. Caracterização sociodemográfica dos participantes $(N=21)$

\begin{tabular}{llcc}
\hline \multicolumn{1}{c}{ DADOS } & \multicolumn{1}{c}{ SOCIODEMOGRÁFICOS } & $\mathrm{N}$ & $\%$ \\
\hline Sexo & Feminino & 13 & 61.9 \\
& Masculino & 8 & 38.0 \\
\hline Faixa etária & $20-30$ & 3 & 14.2 \\
& $31-40$ & 7 & 33.3 \\
& $41-50$ & 5 & 23.8 \\
& $51-60$ & 6 & 28.5 \\
\hline Escolaridade & Ensino Superior & 18 & 85.7 \\
& Ensino Médio & 3 & 14.2 \\
\hline Função na escola & Professor & 15 & 71.4 \\
& PMEC & 1 & 4.7 \\
& Agente de Organização Escolar (AOE) & 3 & 14.2 \\
& Coordenadora pedagógica & 1 & 4.7 \\
& Administrativo & 1 & 4.7 \\
\hline
\end{tabular}

\section{Instrumento}

Foi utilizado o Questionário para Diagnóstico do Meio Escolar (DME) desenvolvido e utilizado em Portugal (Nunes et al., 2015). Após a autorização e adaptação linguística para o português do Brasil, foi feito o teste piloto com três profissionais da rede pública estadual de educação básica.

O DME é um questionário de autopreenchimento constituído de 4 partes: dados sociodemográficos; informações sobre os espaços da escola e o seu entorno; funcionamento da escola, interações e atividades extracurriculares realizadas com outras instituições e comunidade; características e comportamentos dos estudantes (Nunes et al., 2015). O instrumento possui itens com diferentes formatos de resposta (fechada ou aberta). O tempo de aplicação é de 15 minutos. 


\section{Procedimentos}

A coleta de dados foi realizada em maio de 2017, após aprovação do Comitê de Ética e Pesquisa da Escola de Enfermagem de Ribeirão Preto da Universidade de São Paulo (EERP/USP) sob Processo n²034500/2017. Em dia e horário agendados foram apresentados aos participantes os objetivos da pesquisa e solicitado a assinatura do Termo de Consentimento Livre e Esclarecido (TCLE). Os questionários (DME), sem a identificação, foram entregues aos participantes e combinado o prazo para a sua devolução.

Para o tratamento dos dados foi construída, primeiramente e para uma melhor observação das respostas às categorias previamente definidas, uma planilha no $\mathrm{Mi}$ crosoft Excel, versão 2013. As justificativas emergentes das questões abertas foram submetidas a análise temática (Braun \& Clarke, 2006) e consequente obtenção de categorias apresentadas nos resultados.

\section{Resultados}

\section{Ambiente da escola e seu entorno}

Quanto à organização/qualidade dos espaços da escola (biblioteca, cantina, salas de aula e recreio), 9 dos participantes (4 professores, 1 PMEC, 3 agentes de organização escolar -AOE e 1 coordenadora) responderam como sendo bom, também apontaram que os espaços eram bem equipados/organizados e com espaços fundamentais (biblioteca, sala de aula, sala de professores, ginásio ou quadra de esportes). Os espaços foram considerados razoáveis por 5 dos profissionais (4 professores e 1 administrativo).

Por outro lado, 7 dos participantes classificaram como mau (5) e muito mau (2) a organização/qualidade dos espaços da escola. Na justificativa para esta classificação, os participantes apontaram falta de funcionários, de manutenção e limpeza (asseio), carência de equipamentos, de organização e de espaços como biblioteca, sala de aula, sala de professores, ginásio ou quadra de esportes e prática de educação física (ex.: "Falta inspetor e material escolar"; "Não é utilizada a biblioteca, apesar de ter, e nem é incentivado o uso. A escola não dispõe de suporte do governo do Estado para nada. É um faz de conta por parte do governo").

Sobre a adequação das condições estruturais da escola para o número de alunos que possui, 11 dos participantes (8 professores e $3 \mathrm{AOE}$ ) classificaram como sendo razoável. Dos demais, 7 (5 professores, 1 PMEC e 1 coordenadora) consideraram a estrutura da escola como boa e 3 (2 professores e 1 administrativo) a avaliaram como mau.

Em relação às características do entorno da escola 12 dos participantes apontaram estabelecimentos comerciais/industriais, que poderiam ser perturbadores do 
desempenho dos estudantes (bares, cafés e salões de jogos). Além das opções apresentadas no DME, 5 dos participantes (3 professores, 1 PMEC e 1 AOE) apontaram a presença de local de oferta de drogas no entorno da instituição escolar, como aspecto perturbador. A forma como cada um destes se referiu a tal local foi: "boca de fumo", "tráfico de drogas", "pontos de drogas", "ponto de tráfico", "ponto de vendas de drogas".

Houve também a classificação razoável das características do entorno escolar (transporte público, lanchonetes e comércios específicos) por 11 dos participantes (10 professores e 1 administrativo) (ex.: "A escola está inserida dentro de uma média, entre as opções. Nem ruim e nem bom. É razoável, pois atende algumas necessidades”; "Razoável, uma vez que a escola conta com acessos rápidos e posto de saúde").

As características do ambiente, bairro e comunidade em que a escola está inserida foi avaliada como sendo bom por 7 dos participantes (3 professores, 1 PMEC, 2 AOE e 1 coordenador), também apontaram que a escola dispunha de bons acessos, estava bem servida de transportes, bem inserida na comunidade e contava com serviços fundamentais para o seu funcionamento (lanchonete e papelaria). Em contrapartida, 2 professores e 1 AOE classificaram essas características como mau e muito mau, responderam que a escola estava localizada em região periférica/isolada, em ambiente/ comunidade pobre e com segurança precária ou sem segurança.

Neste estudo, os participantes apontaram medidas que poderiam melhorar a escola (cf. Tabela 2).

Tabela 2. Medidas apontadas pelos participantes que poderiam melhorar a escola $(\mathrm{N}=21)$

\begin{tabular}{lccc}
\hline \multicolumn{1}{c}{ Medidas para melhorar a escola } & $\mathrm{n}$ & $\%$ \\
\hline Promover ou ampliar/melhorar o policiamento no entorno da escola & 15 & 71.4 \\
Criar espaços de lazer/esportes & 13 & 61.9 \\
Aumentar número de profissionais para supervisionar a escola & 13 & 61.9 \\
Melhorar as condições da estrutura física da escola & 9 & 42.9 \\
Aumentar a monitorização dos alunos nos intervalos & 6 & 28.5 \\
Aumentar o controle das saídas/entradas na escola & 4 & 19.0 \\
\hline
\end{tabular}

É possível assinalar que o policiamento nas imediações da escola foi a medida mais apontada pelos participantes, seguida da criação de espaços de lazer/esportes e aumento do número de profissionais para supervisionar a escola (cf. Tabela 2). Além dessas medidas, 2 participantes (1 professor e 1 AOE) descreveram outras sugestões: "Colocar em uso os espaços que a escola possui como a sala de informática"; "Se o governo disponibilizasse material humano com condições físicas, mas não. A escola não dispõe de suporte do governo". 


\section{Funcionamento interno e externo da escola}

Quanto as atividades extracurriculares desenvolvidas na escola (eventos, espaços de debate e/ou convivência), 9 dos participantes (6 professores, 1 PMEC, 1 administrativo e $1 \mathrm{AOE}$ ) responderam como sendo razoáveis. Em contrapartida, 8 dos participantes (7 professores e 1 AOE) classificaram essas atividades como mau. $\mathrm{Na}$ justificativa para esta classificação, os participantes alegaram problemas na divulgação e na participação/motivação (ex.: "A escola faz atividades com recursos próprios e com a boa vontade de alguns"; "No caso da escola pública e na atual situação, não há verbas para eventos").

Os participantes apontaram os acontecimentos extracurriculares que regularmente promoviam o envolvimento conjunto de todos (estudantes, professores, funcionários e pais) (cf. Tabela 3). Além das opções apresentadas no DME, 2 dos participantes (1 professor e $1 \mathrm{AOE}$ ) apontaram também dificuldades para o desenvolvimento desses acontecimentos (ex.: "Fazemos o que podemos. O governo não investe em EDUCAÇÃO"; "São muito raros os passeios").

Tabela 3.Acontecimentos extracurriculares que promoviam o envolvimento conjunto de todos $(\mathrm{N}=21)$

\begin{tabular}{lcc}
\hline \multicolumn{1}{c}{ Acontecimentos extracurriculares } & $\mathrm{n}$ & $\%$ \\
\hline Eventos culturais (cinema, teatro) & 20 & 95.2 \\
$\begin{array}{l}\text { Visitas a museus e outros locais históricos } \\
\text { (parques, jardins, monumentos, bibliotecas) }\end{array}$ & 12 & 57.1 \\
$\begin{array}{l}\text { Atividades esportivas } \\
\text { Ações de formação/sensibilização (prevenção) }\end{array}$ & 7 & 33.3 \\
$\begin{array}{l}\text { Atividades lúdicas fora da escola/externas } \\
\text { (parques, jardins, museus, espaços públicos) }\end{array}$ & 5 & 23.8 \\
\hline
\end{tabular}

No que se refere aos acontecimentos potenciais para promover o envolvimento conjunto de todos (estudantes, professores, funcionários e pais) os profissionais destacaram: eventos culturais (cinema, teatro), seguido de visitas à museus e outros locais históricos (parques, jardins, monumentos e bibliotecas) e atividades esportivas (cf. Tabela 3).

Quanto a iniciativa da escola para estabelecer parcerias com outras instituições (outras escolas, universidades, associações/instituições locais), 11 dos participantes (7 professores, 2 AOE, 1 PMEC e 1 administrativo) responderam como sendo razoáveis (ex.: "A única pareceria é com a USP, através dos estagiários, os estudantes desta escola participam de atividades ligadas à saúde"). Para 5 dos participantes (4 professores e 1 administrativo) e 1 professor, essa iniciativa era boa e muito boa. Na justificativa 
para estas classificações, os profissionais apontaram a escola como proativa, contava com diversas parcerias, era conhecedora do meio e das necessidades da comunidade. Em contrapartida, 4 dos participantes (3 professores e 1 AOE) avaliaram como sendo mau essa iniciativa e também apontaram que a escola tinha pouca motivação e faltava recursos financeiros.

Indagados sobre os acontecimentos/eventos extracurriculares que implicavam na parceria entre a escola e outras instituições, os participantes assinalaram: eventos culturais (20), projetos comunitários (8), atividades esportivas (7), protocolos de estágios (curriculares e/ou profissionais) (7), atividades relacionadas com a futura carreira dos estudantes (6) e formação e aprimoramento (capacitação/qualificação) de profissionais de ensino (4). Os participantes apontaram as intuições que tinham parceria com a escola: prefeitura, polícia, igreja, associações/instituições locais, unidades de saúde e outros estabelecimentos de ensino.

Em relação ao envolvimento dos pais nas diferentes atividades escolares, 18 dos participantes classificaram como baixo (8 professores e 1 AOE) e muito baixo (7 professores, 1 administrativo e 1 AOE). Alegaram como justificativas a desmotivação/ desinteresse dos pais, falta de tempo e de divulgação das atividades promovidas pela escola (ex.: "Falta conscientização dos pais"). Houve também a classificação razoável por 3 participantes (1 PMEC, 1 coordenadora e 1 AOE) desse envolvimento.

Sobre o envolvimento comunitário da escola, 15 dos participantes classificaram como sendo baixo (11 professores) e muito baixo (3 professores e $1 \mathrm{AOE}$ ). Apontaram o envolvimento esporádico, falta de apoio/interesse da comunidade e falta de adesão/ divulgação como justificativas. A avaliação razoável do envolvimento comunitário foi apontada por 5 dos participantes (1 professor, 1 administrativo, 1 coordenadora, 1 PMEC e 1 AOE). Apenas um AOE considerou elevado este envolvimento, alegando a participação em projetos comunitários.

O sistema de medidas disciplinares adotado na escola foi considerado bom por 9 dos participantes (5 professores, 1 PMEC, 1 administrativo, 1 coordenadora pedagógica e 1 AOE). Na justificativa para esta classificação, os participantes apontaram que a atuação é imediata, as medidas eram aplicadas com eficácia e apropriadas aos comportamentos demonstrados. As medidas disciplinares também foram consideradas como razoável por 7 dos participantes (5 professores e 2 AOE). Em contrapartida, 5 dos participantes, sobretudo professores, responderam como sendo mau esse sistema e apontaram a falta de critérios na sua aplicação e a atuação demorada (ex.: "Escola mal organizada, devido à falta de funcionários").

Os participantes apontaram medidas que poderiam melhorar o sistema disciplinar da escola (cf. Tabela 4). 
Tabela 4.Medidas apontadas pelos participantes para melhorar o sistema disciplinar da escola $(\mathrm{N}=21)$

\begin{tabular}{|c|c|c|}
\hline MEDIDAS PARA MELHORAR O SISTEMA DISCIPLINAR & $\mathrm{N}$ & $\%$ \\
\hline Promover o envolvimento dos pais/responsáveis dos estudantes & 18 & 85.7 \\
\hline $\begin{array}{l}\text { Criar equipes multidisciplinares (psicólogos, enfermeiros, } \\
\text { profissionais do direito, assistentes sociais, sociólogos) }\end{array}$ & 13 & 61.9 \\
\hline Aumentar número de profissionais do ensino & 12 & 57.1 \\
\hline Reduzir o número de estudantes por turma/escola & 9 & 42.8 \\
\hline Tornar o sistema disciplinar mais rigoroso & 8 & 38.0 \\
\hline Aumentar a vigilância no recreio & 5 & 23.8 \\
\hline $\begin{array}{l}\text { Criar horários diferenciados para o tempo de recreio } \\
\text { atendendo a faixa etária da turma }\end{array}$ & 2 & 9.5 \\
\hline
\end{tabular}

Pode-se observar que uma parte expressiva dos participantes (18) acreditava que promover o envolvimento dos pais/responsáveis dos estudantes poderia melhorar o sistema disciplinar da escola, seguido de criar equipes multidisciplinares e aumentar o número de profissionais de ensino (cf. Tabela 4).

Indagados sobre as áreas de conhecimento ou profissional que consideravam pertinentes para melhorar o sistema disciplinar da escola, os participantes responderam: psicologia (17), serviço social (16), sociologia (6) e enfermagem (8). Foi apresentado pelo PMEC no item aberto para outras opções a filosofia.

Em relação as ações consideradas úteis para os participantes desenvolverem na escola, foram apontadas: apoio psicológico para a comunidade (18), ações de sensibilização/informação (19), orientação vocacional dos estudantes (11) e identificar e intervir em diversas problemáticas (econômicas, socioculturais) (11).

\section{Características e comportamentos dos estudantes}

Quanto ao comportamento dos estudantes da escola, 10 dos participantes (7 professores, 2 AOE e 1 coordenadora) e 1 professor responderam como sendo mau e muito mau. Na justificativa para esta classificação, os participantes apontaram que os estudantes manifestavam comportamentos inadequados em sala de aula e recreio, havia falta de modelos parentais e os estudantes não tinham respeito pela autoridade no contexto escolar (ex.: "Em relação ao comportamento temos que levar em conta as condições sociais, econômicas e culturais que interferem. O meio com a genética também"; "Muita agressividade entre eles"). Houve também a avaliação razoável do comportamento dos estudantes por 8 dos participantes (6 professores, 1 administrativo e 1 PMEC) e 2 dos participantes (1 professor e 1 AOE) classificaram como sendo bom, alegando casos pontuais de mau comportamento. 
Nesse estudo, os participantes apontaram alguns dos principais problemas de comportamento dos estudantes na escola (cf. Tabela 5).

Tabela 5.Problemas de comportamento apontados pelos participantes $(\mathrm{N}=21)$

\begin{tabular}{lcc}
\hline \multicolumn{1}{c}{ PROBLEMAS DE COMPORTAMENTO DOS ESTUDANTES } & $\mathrm{N}$ & $\%$ \\
\hline Desrespeito pelos colegas & 17 & 80.9 \\
Desrespeito pelos professores & 13 & 61.9 \\
Desrespeito pelos funcionários & 13 & 61.9 \\
Desrespeito generalizado & 13 & 61.9 \\
Manifestação de comportamentos agressivos & 13 & 61.9 \\
Consumo de tabaco/drogas & 8 & 38.0 \\
Consumo de álcool & 2 & 9.5 \\
\hline
\end{tabular}

Nota-se que o desrespeito entre os estudantes é apontado pelos participantes como principal problema de comportamento na escola, seguido do desrespeito pelos professores e funcionários, desrespeito generalizado e manifestação de comportamentos agressivos (cf. Tabela 5).

Tabela 6. Incivilidades apontadas pelos participantes $(\mathrm{N}=21)$

\begin{tabular}{lcc}
\hline \multicolumn{1}{c}{ INCIVILIDADES } & $\mathrm{N}$ & $\%$ \\
\hline Utilização de linguagem imprópria & 20 & 95.2 \\
Perturbar o funcionamento escolar com ruídos, & 17 & 80.9 \\
correrias, gritos, música alta & 16 & 76.1 \\
Destruir/danificar equipamentos & 9 & 42.8 \\
Espalhar/jogar lixo pela escola & & \\
\hline
\end{tabular}

A utilização de linguagem imprópria é uma das incivilidades mais frequentes entre os estudantes, seguida de perturbar o funcionamento escolar e destruir/danificar equipamentos (cf. Tabela 6).

Questionados se o comportamento dos estudantes tem se agravado nos últimos 5 anos, 15 dos participantes (13 professores, 1 administrativo e 1 AOE) responderam afirmativamente. Esse agravamento foi relacionado pelos profissionais da amostra, sobretudo, aos problemas no contexto familiar, problemas socioeconômicos e utilização das redes sociais de forma inadequada (cf. Tabela 7). 
Tabela 7.Fatores relacionados ao agravamento do comportamento dos estudantes ( $N=21)$

\begin{tabular}{lcc}
\hline \multicolumn{1}{c}{ FATORES DE RISCO } & $\mathrm{N}$ & $\%$ \\
\hline Problemas no contexto familiar & 14 & 66.6 \\
Problemas socioeconômicos/desemprego & 13 & 61.9 \\
Utilização das redes sociais de forma inadequada & 10 & 47.6 \\
Ausência de motivação dos alunos & 9 & 42.8 \\
Reduzido controle parental & 7 & 33.3 \\
Elevado número de alunos por turma/escola & 7 & 33.3 \\
Consumo abusivo de substâncias & 7 & 33.3 \\
Utilização de jogos de computador/videogames & 3 & 14.2 \\
Inadequação do regulamento escolar & 1 & 4.7 \\
\hline
\end{tabular}

Por outro lado, 6 dos participantes (2 professores, 2 AOE, 1 PMEC e 1 coordenadora) responderam negativamente para o agravamento do comportamento dos estudantes nos últimos 5 anos.

A responsabilização dos pais, seguida da intervenção/prevenção focada em diferentes ocorrências e reforço dos recursos humanos foram medidas apontadas para combater/prevenir os comportamentos menos adequados dos estudantes (cf. Tabela 8).

Tabela 8. Medidas para combater/prevenir os comportamentos menos adequados dos estudantes na escola $(\mathrm{N}=21)$

\begin{tabular}{lcc}
\hline \multicolumn{1}{c}{ MEDIDAS } & $\mathrm{N}$ & $\%$ \\
\hline Maior responsabilização dos pais dos estudantes & 19 & 90.4 \\
Intervenção/prevenção focada em diferentes ocorrências & 15 & 71.4 \\
Reforço dos recursos humanos & 13 & 61.9 \\
Maior autoridade/importância da escola & 11 & 52.3 \\
Reduzir o número de estudantes por turma/escola & 9 & 42.8 \\
Maior rigor/exigência dos profissionais & 7 & 33.3 \\
\hline
\end{tabular}

\section{Discussão}

Os profissionais do nosso estudo foram, sobretudo professores. Essa participação expressiva está relacionada ao fato de o número deste profissional na escola ser consideravelmente superior ao restante dos funcionários, segundo resultados de estudo realizado em Portugal (Nunes et al., 2015)

Os participantes, de modo geral, revelam percepções satisfatórias em relação as condições estruturais da escola. Contudo, a instituição escolar não se caracteriza apenas pelos espaços físicos, mas também pelos profissionais que integram esse 
ambiente, sua comunidade e sobretudo os materiais, equipamentos e elementos necessários para o seu funcionamento (Monteiro \& Da Silva, 2015). Neste estudo, foi identificado que o entorno da escola tem características que pode afetar o processo educativo dos estudantes como a vulnerabilidade social e econômica, incluindo o tráfico de drogas. De acordo com Érnica e Batista (2012), quanto maiores os níveis de vulnerabilidade social do entorno da escola, maior a restrição na qualidade do ensino oferecido. Além disso, a violência que ocorre no entorno da escola continua a ser um grande problema de saúde pública entre os atores escolares, uma vez que esta instituição tem sido afetada pela extensão da violência urbana contemporânea, incluindo o narcotráfico e o crime (Becker \& Kassouf, 2016).

A amostra é unânime quanto a carência de investimento do governo na educação. No Brasil, a negligência do poder público é um fenômeno histórico que acomete a educação, infligindo os direitos dos estudantes e dos profissionais. De modo que as políticas nacionais da educação contribuem para a precarização do ensino público, resultando nos baixos índices de desempenho dos estudantes e promovendo a exclusão social (De Souza, 2016; Libâneo, 2016). Cenário preocupante visto a importância da escola e da educação pública de qualidade para a sociedade.

Nesta investigação também se verificam o baixo envolvimento dos pais e da comunidade com a escola. Na perspectiva de Vieira e Vidal (2015), a relação entre escola-família-comunidade é afetada pelas políticas públicas brasileiras que são concebidas numa visão de totalidade, não considerando as peculiaridades dos diferentes entornos da escola, tão pouco as especificidades das violentas periferias urbanas, tornando-se assim distante a relação entre os atores escolares.

Perante a análise, os participantes parecem acreditar que o sistema disciplinar da escola pode ser melhorado promovendo a participação dos pais. Um estudo sobre o nível de participação dos pais na escola, mostrou que este envolvimento influencia o desenvolvimento educativo dos estudantes, tendo como propósito a continuidade do trabalho em casa e ampliação das situações de aprendizagem. Os resultados mostram que, desse modo, os estudantes são incentivados a alcançarem resultados mais positivos, tornando-se mais interessados, felizes e responsáveis, valorizando a escola e os professores (Bento, Mendes, \& Pacheco, 2016). No entanto, é preciso ter cuidado ao fazer essa referência, uma vez que o estigma do fracasso escolar tende a perseguir as crianças oriundas dos extratos sociais mais empobrecidos da população, uma vez que é relacionado o sucesso escolar dos estudantes como dependente da participação da família e da comunidade (Vieira \& Vidal, 2015).

A atuação de equipe multidisciplinar foi considerada pelos participantes como importante para o bom funcionamento da escola. De fato, a atuação do psicólogo, assistente social, enfermeiro dentre outros profissionais podem favorecer o processo educativo do estudante, pois, além de promover a articulação entre os diferentes saberes 
no intuito de melhorar a qualidade das relações, também viabiliza ações intersetoriais, promovendo o cuidado integral de crianças e adolescentes (Da Silva \& Assis, 2017).

No que se refere ao comportamento dos estudantes, os profissionais da amostra percebem seu agravamento nos últimos cinco anos, houve a manifestação de atos de incivilidade, desrespeito pelos colegas e manifestação de comportamento agressivo. Estudos mostram que o clima escolar negativo está diretamente relacionado com a manifestação da violência como, por exemplo, a vitimização do bullying, comparado as escolas com clima mais positivo (Konold, Cornell, Shukla, \& Huang, 2017; Shukla, Konold, \& Cornell, 2016; Yang, Sharkey, Reed, Chen, \& Dowdy, 2018). De acordo com Silva e Costa (2016), as escolas que adotam medidas disciplinares punitivas ou que possui professores com comportamento agressivo, tornam-se ambientes de constante tensão e conturbação, induzindo os estudantes à manifestação de comportamentos violentos.

No entanto, os profissionais da amostra atribuem o agravamento do comportamento dos estudantes, sobretudo aos problemas no contexto familiar e às questões socioeconômicos/desemprego. Essas questões, de fato, estão relacionadas ao desempenho acadêmico do estudante, contudo, estudos evidenciam a importância do clima escolar positivo para que os atores escolares se sintam engajados e respeitados, promovendo um ambiente de aprendizagem social, emocional e ético (Konold et al., 2017; Shukla et al., 2016; Yang et al., 2018). De modo que para o bom comportamento e desempenho do estudante é fundamental o envolvimento dos atores escolares, tendo ciência das regras e normas, sem imposições, na perspectiva de horizontalidade e não de autocracia (Salgado-Junior, Novil, \& Ferreira, 2017).

Nesta investigação também se verificam as medidas que poderiam melhorar o funcionamento escolar como a construção de espaços de lazer/esportes, promoção de eventos culturais, policiamento e aumento do número de profissionais para supervisionar a escola. Para implementação dessas medidas é importante promover a articulação intersetorial, considerando também a participação da família e da comunidade. Nesse sentido, é possível o desenvolvimento de ações que integram diferentes serviços como da saúde, da educação, de esporte, da cultura, da assistência social, de segurança pública dentre outros (Espelage, Polanin, \& Low, 2014; De Sousa, Esperidião, \& Medina, 2017).

\section{Conclusão}

$\mathrm{Na}$ opinião dos profissionais que participaram deste estudo, as principais dificuldades que a escola precisa enfrentar, porque afetam seu funcionamento, são: a falta de recursos; falta de envolvimento dos estudantes, da família e da comunidade; manifestação de atos de incivilidade e comportamento agressivo. Embora, tenhamos 
uma amostra específica e um número reduzido de profissionais, destacamos a partir desse debate empírico, que fazer o diagnóstico do meio escolar com informações dos profissionais que atuam nas escolas é necessário, pois, oferece subsídios para o desenvolvimento de ações que visem o gerenciamento de comportamentos, a prevenção e enfrentamento da violência escolar. Sugerimos a realização de mais estudos em número maior de instituições de ensino que considerem as especificidades da escola e sua comunidade, tendo como propósito o desenvolvimento de estratégias que possam contribuir para o funcionamento escolar.

Observação: Este artigo enquadra-se no âmbito da tese de doutoramento "Pesquisa-ação e práticas restaurativas para a prevenção da violência escolar: percepções dos professores", desenvolvida pelo Programa de Pós-Graduação em Enfermagem Psiquiátrica na Escola de Enfermagem de Ribeirão Preto da Universidade de São Paulo, Centro Colaborador da OPAS/OMS para o Desenvolvimento da Pesquisa em Enfermagem, Ribeirão Preto, SP, Brasil.

\section{Referências}

Becker, K. L., \& Kassouf, A. L. (2016). Violência nas escolas públicas brasileiras: Uma análise da relação entre o comportamento agressivo dos alunos e o ambiente escolar. Nova Economia, 26(2), 653-677.

Bento, V. A., Mendes, R. G., \& Pacheco, D. (2016). Relação escola-família: participação dos encarregados de educação na escola. Investigação Qualitativa em Educação, 1, 603-612.

Bernardes, T. A. (2015). Hegemonia neoliberal, precarização do trabalho docente no ensino público do Estado de São Paulo e adoecimento - uma análise a partir de Ourinhos - SP. Revista Pegada, 16(especial), 29-40.

Brasil. (2013). Ministério da Educação. Secretária de Educação Básica. Secretária de Educação Continuada, Alfabetização, Diversidade e Inclusão. Diretrizes curriculares nacionais da educação básica. Diretoria de Currículos e Educação Integral. Brasília.

Braun, V., \& Clarke, V. (2006). Using thematic analysis in psychology. Qualitative Research in Psychology, 3(2), 77-101.

Brenner, A. K., \& Carrano, P. C. R. (2014). Os sentidos da presença dos jovens no ensino médio: representações da escola em três filmes de estudantes. Educação e Sociedade, 35(129), 12231240.

Da Silva, R. F., \& Assis, G. S. (2017). Prevenção da violência escolar: uma revisão da literatura. Educação e Pesquisa, Ahead of print. [Disponível em http://dx.doi.org/10.1590/S1517-9702201703157305, consultado em 04/04/2018].

De Souza, M. C. R. F. (2016). Estudantes em tempo integral no campo: aprendizagem, processos e sentidos. Cadernos de Pesquisa, 46(161),756-782.

De Sousa, M.C., Esperidião, M. A., \& Medina, M. G. (2017). A intersetorialidade no Programa Saúde na Escola: avaliação do processo político-gerencial e das práticas de trabalho. Ciência \& Saúde Coletiva, 22(6), 1781-1790. 
Érnica, M., \& Batista, G. A. A. (2012). A escola, a metrópole e a vizinhança vulnerável. Cadernos de Pesquisa, 42(146), 640-666.

Espelage, D. L., Polanin, J. R., \& Low, S. K. (2014). Teacher and staff perceptions of school environment as predictors of student aggression, victimization, and willingness to intervene in bullying situations. School Psychology Quarterly, 29(3), 287-305.

Konold, T., Cornell1, D., Shukla, K., \& Huang, F. (2017). Racial/Ethnic Differences in Perceptions of School Climate and Its Association with Student Engagement and Peer Aggression. Journal of Youth and Adolescence, 46, 1289-1303.

Libâneo, J. C. (2016). Políticas educacionais no Brasil: desfiguramento da escola e do conhecimento escolar. Cadernos de Pesquisa, 46(159), 38-62.

Martins, A. M., \& Alves, M. G. (2018). Situações de conflito e violência em escolas públicas: Aproximações entre Brasil e Portugal. Revista de Sociología de la Educación, 11 ( I), 153-166.

Monteiro, S. J., \& Da Silva, P. D. (2015). A influência da estrutura escolar no processo de ensinoaprendizagem: uma análise baseada nas experiências do estágio supervisionado em Geografia. Geografia Ensino \& Pesquisa, 19(3), 19-28.

Nunes, L. M., Caridade, S., \& Sani, A. I. (2015). Avaliação do meio escolar: um estudo exploratório. Revista Lusófona de Educação, 30, 141-158.

Ollaik, L. G., \& Ziller, H. M. (2012). Concepções de validade em pesquisas qualitativas. Educação e Pesquisa, 38(1), 229-241.

Salgado-Junior, A. P., Novil, J. C. \& Ferreira, J. (2016). Práticas escolares e desempenho dos alunos: uso das abordagens quantitativa e qualitativa. Educação e Sociedade, 37(134), 217-243.

Scherer, P. A. Z., Scherer, A. E., Cocenas, A. S., \& Gregorio, S. A. (2011). Grupo de atividades estruturadas com estudantes: identificação de violência no ambiente escolar. Revista Cultura e Extensão USP, 6, 111-119.

Shukla, K., Konold, T., \& Cornell, D. (2016). Profiles of Student Perceptions of School Climate: Relations with Risk Behaviors and Academic Outcomes. American Journal of Community Psychology, 57, 291-307.

Silva, S. S., \& Costa, B. L. D. (2016). Opressão nas escolas: o Bullying entre estudantes do ensino básico. Cadernos de Pesquisa, 46(161), 638-663.

Vieira, S. L., \& Vidal, E. M. (2015). Gestão democrática da escola no brasil: desafios à implementação de um novo modelo. Revista Iberoamericana de Educación, 67, 19-38.

Yang, C., Sharkey, J. D., Reed, L. A., Chen, C., \& Dowdy, E. (2018). Bullying victimization and student engagement in elementary, middle, and high schools: Moderating role of school climate. School Psychology Quarterly, 33(1), 54-64 [Disponível em http://dx.doi.org/10.1037/spq0000250, consultado em 04/04/2018]. 


\section{Jaqueline Rodrigues Stefanini} PhD, Professora da Faculdade Unida de Campinas - Goiânia - Brasil

E-mail: jaquestefanini@gmail.com ORCID: http://orcid.org/0000-0002-2495-9668

\section{Luciana Aparecida Cavalin}

Doutoranda, Psicóloga, Instituto Federal de Educação, Ciência e Tecnologia.

São Paulo -Brasil.

Email: lucianacavalin@usp.br; ORCID: http://orcid.org/0000-0002-3537-9028

\section{Débora Tatiane Góes Silva}

Mestre, Escola de Enfermagem de Ribeirão Preto,

Universidade de São Paulo,

Centro Colaborador da OPAS/OMS

para o Desenvolvimento da Pesquisa em Enfermagem, Ribeirão Preto, SP, Brasil

Email: deboragsilva@usp.br

ORCID: http://orcid.org/0000-0002-6593-6702

Natalia Alves de Camargo

Mestranda, Escola de Enfermagem de Ribeirão Preto,

Universidade de São Paulo, Centro Colaborador da OPAS/OMS para o Desenvolvimento da Pesquisa em Enfermagem, Ribeirão Preto, SP, Brasil -

Email: nataliacamargo@usp.br ORCID: http://orcid.org/0000-0001-8326-2986

Ana Isabel Sani PhD, Professora da Universidade Fernando Pessoa Porto-Portugal Email: anasani@ufp.edu.pt; ORCID: http://orcid.org/0000-0003-1776-2442

\section{Edson Arthur Scherer}

$\mathrm{PhD}$, Psiquiatra, Hospital das Clínicas, Faculdade de Medicina de Ribeirão Preto, Universidade de São Paulo, Ribeirão Preto, SP, Brasil

Email: eascherer@hcrp.usp.br ORCID: http://orcid.org/0000-0003-0182-6691 


\section{Zeyne Alves Pires Scherer}

$\mathrm{PhD}$, Professora Associada da Escola de Enfermagem de Ribeirão Preto da Universidade de São Paulo, Ribeirão Preto, SP, Brasil

Email: scherer@eerp.usp.br

ORCID: http://orcid.org/0000-0002-3162-5957

Correspondência

Ana Isabel Sani

Universidade Fernando Pessoa

Praça 9 de abril, 349

4249-004 Porto, Portugal

Data de submissão: Março 2018

Data de avaliação: Maio 2018

Data de Publicação: Dezembro de 2019 